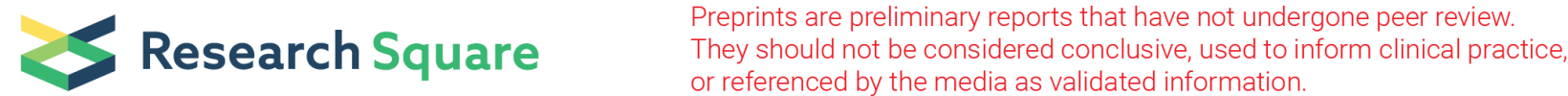

\section{Implication of Retrobulbar and Internal Carotid Artery Blood-Flow-Volume Alterations for the Pathogenesis of Non-arteritic Anterior Ischemic Optic Neuropathy}

\author{
Zhiyong Fu \\ Capital Medical University \\ Hongyang Li \\ Capital Medical University \\ Yanling Wang ( $\sim$ b502367790@126.com ) \\ Capital Medical University
}

\section{Research Article}

Keywords: Blood flow volume, Optical coherence tomography angiography, Pathogenesis, Non-arteritic anterior ischemic optic neuropathy

Posted Date: March 19th, 2021

DOl: https://doi.org/10.21203/rs.3.rs-320300/v1

License: (c) (i) This work is licensed under a Creative Commons Attribution 4.0 International License.

Read Full License 


\section{Implication of Retrobulbar and Internal Carotid Artery Blood-Flow-Volume Alterations for the Pathogenesis of Non-arteritic Anterior Ischemic Optic Neuropathy}

Zhiyong Fu, Hongyang Li, Yanling Wang*

Department of ophthalmology, Beijing Friendship Hospital, Capital Medical University, Beijing 100050, China

*Correspondence: b502367790@126.com

\section{Abstract}

Background: To analyze blood flow volume alteration that involved both retrobulbar artery and internal carotid artery (ICA) in patients with non-arteritic anterior ischemic optic neuropathy (NAION) and to assess their relevance for the pathogenesis of NAION.

Methods: Forty two patients with NAION (unilateral affected) and 42 age-matched controls participated in this study. By head-and-neck computed tomographic angiography (CTA), the diameter of ICA and ophthalmic artery (OA) were measured. By colour Doppler imaging (CDI), the mean blood flow velocity $(\mathrm{Vm})$ and the blood flow volume of ICA and OA were measured or calculated. By optical coherence tomography angiography (OCTA), peripapillary and optic disc vessel density were measured. Data obtained from the affected side of the patients were compared to those of the contralateral healthy side and the control.

Results: Compared with the controls and the contralateral healthy side of the patients with NAION, the diameter of ICA, the blood flow volume of ICA and OA, the peripapillary and optic disc vessel density in the affected side decreased significantly $(p<0.05)$. However, there was no statistical difference in the diameter of $O A(p=0.179,0.054$ respectively), the $\mathrm{Vm}$ of $O A$ ( $p=0.052,0.083$ respectively), or the $\mathrm{Vm}$ of ICA ( $p=0.364,0.938$ respectively) between groups Peripapillary and optic disc vessel density were significantly positive correlated with the blood flow volume in ipsilateral ICA and OA in patients with NAION (all $p<0.01$ ).

Conclusions: The reduction of blood flow volume was more prominent in OA and ICA than 
decrease of $\mathrm{Vm}$, peripapillary and optic disc vessel density were significantly positive correlated with the blood flow volume of ipsilateral ICA and OA in patients with NAION. This finding may imply a predominant affection of retrobulbar artery and ICA blood flow volume alterations in the pathogenesis of NAION.

Keywords: Blood flow volume, Optical coherence tomography angiography, Pathogenesis, Non-arteritic anterior ischemic optic neuropathy

\section{Background}

Non-arteritic anterior ischemic optic neuropathy (NAION) is among the leading causes of optic nerve impairment in the middle-aged and elderly [1,2]. Numerous clinical and experimental studies have been conducted in patients with NAION, which suggested that there was early affection of retrobulbar and carotid artery haemodynamics alterations in the course of the disease [3-6]. Previous study demonstrated that NAION patients showed markedly different retrobulbar haemodynamics with reduced circulation velocity in ophthalmic artery $(\mathrm{OA})$ measured by colour Doppler imaging (CDI) [4]. Furthermore, some researchers suggested that NAION might occur simultaneously with stenosis and reduced blood flow of internal carotid artery (ICA) [7,8]. Due to these reasons, we believed that it was important to assess OA and ICA blood flow while studying the pathogenesis of NAION. Because the siphon region of ICA (ICAS) is often implicated in ocular ischemic diseases and the changes of blood flow here might affect the lower reaches' hemodynamics directly [9], ICAS was designated as the anatomical site for the study of ICA when it was involved.

With the emergence of optical coherence tomography angiography (OCTA), the study of microvasculature of the posterior pole has made great improvement, and this technique sets up a bridge for understanding the relationship between distal macrovascular blood flow volume variations and the developing of NAION. In the present study, we hypothesized that the changes in peripapillary and optic disc vessel density correlate with the blood flow volume alterations in OA and ICA, and aiming to illustrate the importance of evaluating retrobulbar artery and ICA blood flow volume in patients with NAION.

\section{Methods}


A total of 42 patients with unilateral NAION were included in this study, comprising 27 male and 15 female, aged $47-77$ years. The mean interval between onset of NAION and measurement was $5.2 \pm 2.7$ days. All patients met the diagnostic criteria of NAION, including sudden painless loss of visual acuity; presence of optic disc edema shortly after the disease onset; optic disc-related visual field defects; and an absence of other ocular diseases that might affect the optic nerve [10,11]. All patients were clinically diagnosed with NAION after consultating from neurology, infectious diseases, cardiology and rheumatology, in order to exclude any systemic or neurologic diseases that might affect the optic nerve. Patients were excluded if they had serious media opacities or had undergone previous intraocular surgery. The control group consisted of 42 age- matched subjects with no history of chronic ocular disease or of ocular surgery, comprising 25 male and 17 female, aged 49-74 years. Participants were excluded if they had a history of chronic corticosteroids, aspirin, or related drugs use.

This study was approved by the Ethics Committee of Beijing Friendship Hospital affiliated to Capital Medical University and was performed in accordance with the Declaration of Helsinki, and informed consent was obtained from all participants.

Ocular data were collected from all participants, including slit-lamp Ophthalmoscopy, fundus examination, non-contact intraocular pressure (IOP) measurement, fundus photography and spectral-domain optical coherence tomography scans (SD-OCT, Heidelberg, Germany). The Vm of OA and ICA were measured by CDI, and the diameter of OA and ICA were measured by CTA as previously described[12,13]. The diameter of OA was measured by selecting the blood vessel $5 \mathrm{~mm}$ above the level of optic canal outlet and measuring its diameter of (Fig. 1).

30 of 42 patients and 30 of controls also underwent OCTA (OCT-A, Optovue, USA) examination after CDI examinations. The OCTA images were acquired using the Optovue Avanti, and two imaging windows $(4.5 \times 4.5 \mathrm{~mm}$ and $6 \times 6 \mathrm{~mm})$ were obtained. OCTA examination was employed to evaluate peripapillary and optic disc vessel density. Among the four references the system provides, the nerve head mode and RPC mode were used in the present study. The nerve head mode included signals from the inner limiting membrane (ILM) to $150 \mathrm{~mm}$ below the membrane, and the RPC mode included signals from ILM to nerve fiber layer (NFL) (Fig. 2、3).

The blood flow volume (A) of ICA and OA was calculated using the following formula: $\mathrm{A}=\pi \mathrm{r}^{2} \times \mathrm{Vm} \quad(\mathrm{r}=1 / 2$ diameter $)$ 
Blood pressure (BP) was determined by sphygmomanometry in a sitting position after a rest of $10 \min$.

All the measurements were performed by experienced operators unaware of the subject's condition. The left side measurements were defined as the results of each control .

Statistical analyses was conducted via the SPSS statistical package (SPSS, V. 21.0, Chicago, IL, USA).The Kolmogorov-Smirnov test was used to determine the distribution pattern of all obtained data. Continuous variables were compared between groups using two- sample t-test and categorical variables were analyzed by $\chi 2$ test. Pearson correlation coefficient analysis were used to evaluate the associations between peripapillary and optic disc vessel density with the blood flow volume of ICA and OA. The values were presented as means \pm standard deviation (SD) and were considered statistically significant at $\mathrm{P}<0.05$.

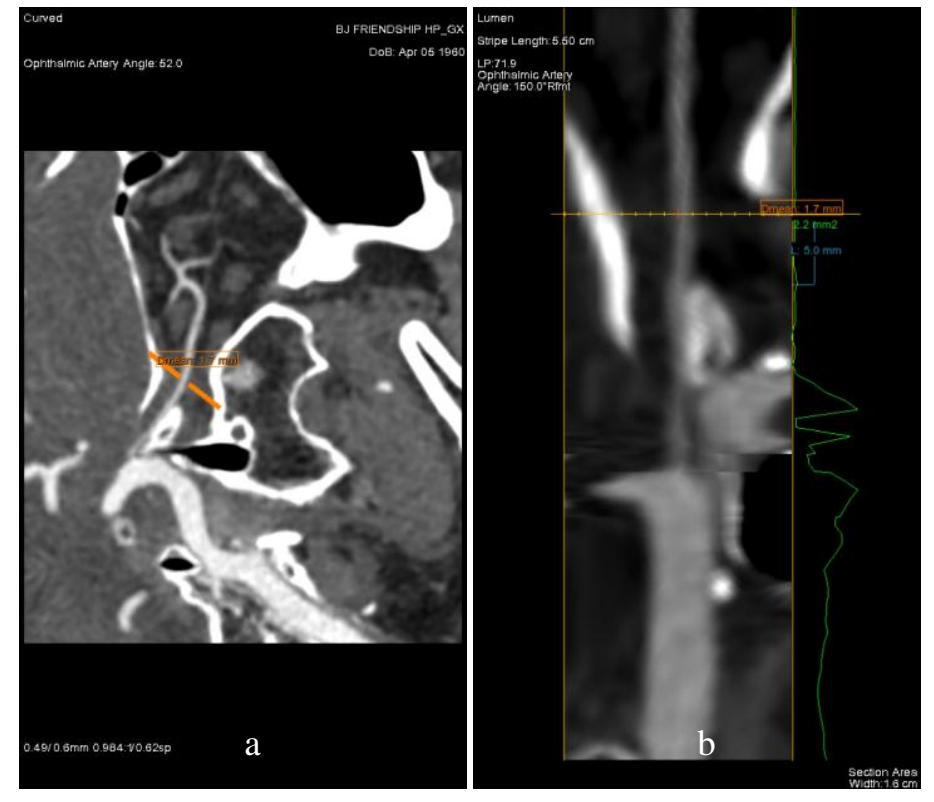

Fig. 1 Head-and-neck CTA inspection. Showed the diameter of OA (a, b )
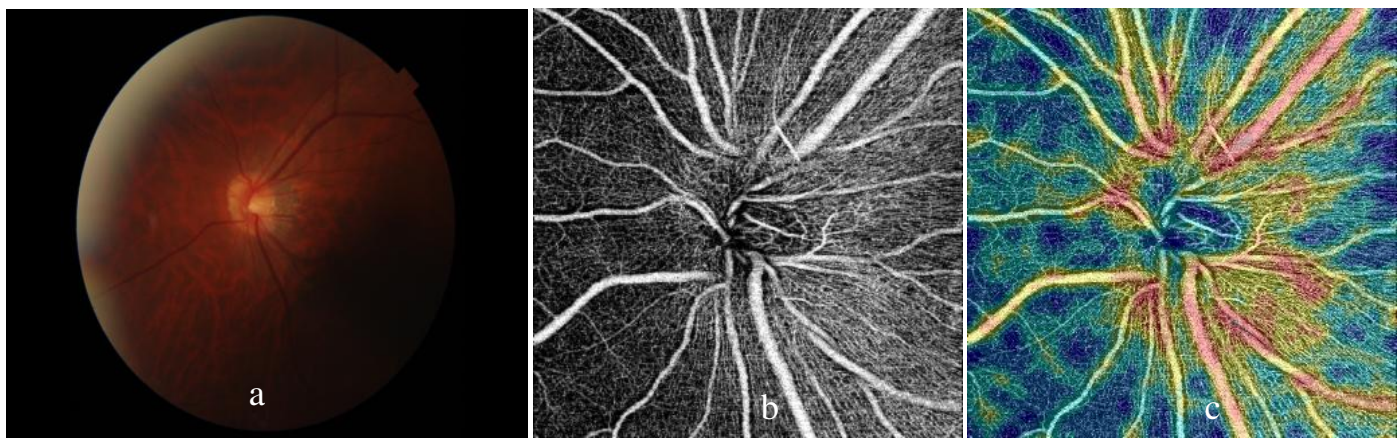


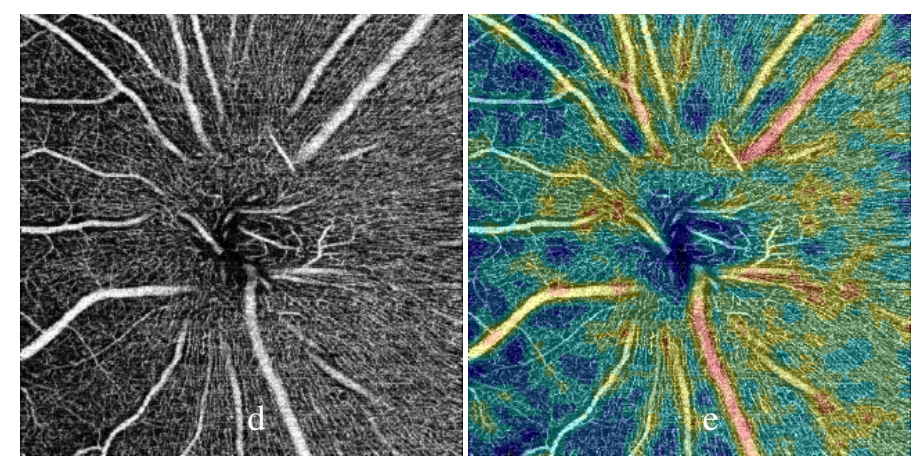

Fig. 2 OCTA examinations of peripapillary and optic disc vessel density of a control subject (left eye). Color fundus photograph (a),vessel density in the peripapillary retina on nerve head mode (b, c) and optic disc on RPC mode (d,e) were included (b, d: images in gray scale, c, e: color-coded images)
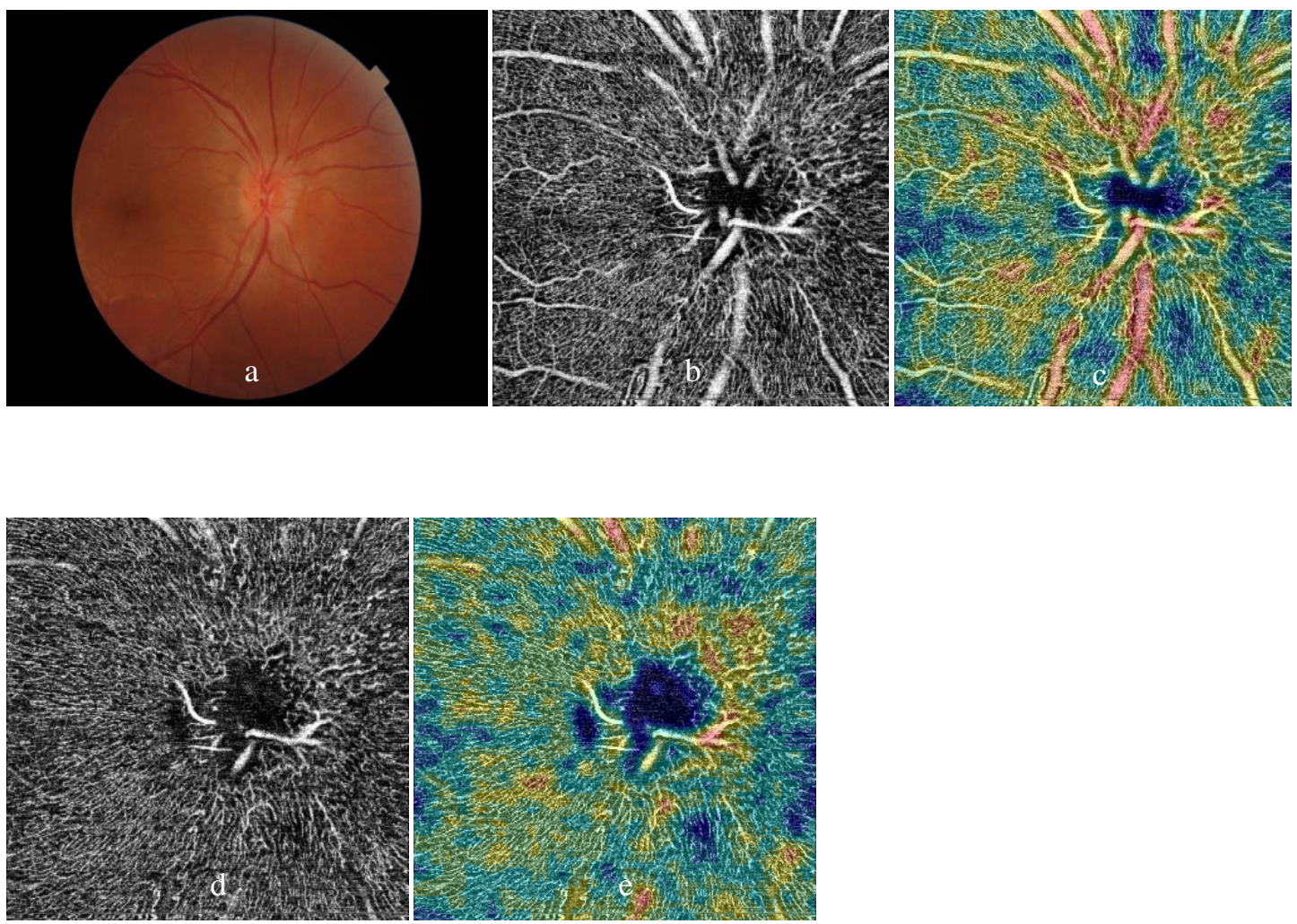

Fig. 3 OCTA examinations of peripapillary and optic disc vessel density of a NAION patient (affected left eye ). Color fundus photograph (a),vessel density in the peripapillary retina on nerve head mode $(b, c)$ and optic disc on RPC mode (d, e) were included (b, d: images in gray scale, c, e: color-coded images)

\section{Results}


Concerning the demographic and clinical data of the NAION patients and the controls, the age, gender composition, IOP, systolic BP (SBP), diastolic BP (DBP) and the proportion of systemic diseases showed no statistically significant difference between two groups（ $>0.05 ）$ (Table 1)

Compared between the affected side and the contralateral healthy side in the patients with NAION, the diameter of ICA, the blood flow volume of ICA, the blood flow volume of OA, peripapillary and optic disc vessel density all significantly decreased $(\mathrm{p}<0.05)$. There was no statistical difference in the diameter of OA $(\mathrm{p}=0.054)$, the $\mathrm{Vm}$ of OA $(\mathrm{p}=0.083)$, or the $\mathrm{Vm}$ of ICA $(\mathrm{p}=0.938) .($ Table 2$)$.

Compared between the affected side of NAION and the controls, the diameter of ICA, the blood flow volume of ICA, the blood flow volume of OA, peripapillary and optic disc vessel density all significantly decreased $(\mathrm{p}<0.05)$. There was no statistical difference in the diameter of OA $(\mathrm{p}=0.179)$, the $\mathrm{Vm}$ of $\mathrm{OA}(\mathrm{p}=0.052)$, or the $\mathrm{Vm}$ of ICA $(\mathrm{p}=0.364)$. (Table 3$)$.

Concerning the correlation between the blood flow volume and vessel density in the affected side of patients with NAION, we found both peripapillary vessel density and optic disc vessel density were significantly positive correlated with the blood flow volume in ipsilateral ICA and OA (all p<0.01) (Table 4).

Table 1 Demographic and clinical data of the patients with NAION and controls

\begin{tabular}{llll}
\hline Groups & NAION & Controls & $P$ \\
\hline Age (years) & $61 \pm 8$ & $60 \pm 7$ & $0.544^{*}$ \\
\hline Gender, no. of male (\%) & $27(64.3 \%)$ & $25(59.5 \%)$ & $0.503^{\star *}$ \\
\hline IOP (mm Hg) & $16 \pm 3$ & $15 \pm 3$ & $0.264^{*}$ \\
SBP (mm Hg) & $138 \pm 23$ & $131 \pm 18$ & $0.134^{*}$ \\
DBP (mm Hg) & $77 \pm 11$ & $73 \pm 9$ & $0.077^{*}$ \\
Hypertension,n (\%) & $13(31.0 \%)$ & $10(23.8 \%)$ & $0.268^{* *}$ \\
Diabetes mellitus,n (\%) & $11(26.2 \%)$ & $10(23.8 \%)$ & $0.744^{* *}$ \\
Hyperlipidemia,n (\%) & $20(47.6 \%)$ & $15(35.7 \%)$ & $0.086^{* *}$ \\
Ischaemic heart diseas,n & $5(11.9 \%)$ & $3(7.1 \%)$ & $0.228^{* *}$ \\
(\%) & & & \\
\hline
\end{tabular}

Independent-samples t-test ${ }^{*}, \mathrm{X} 2$ test ${ }^{* *}$, Paired- samples t-test ${ }^{* * *}$ 
NAION non-arteritic anterior ischemic optic neuropathy, IOP intraocular pressure, SBP systolic blood pressure, DBP diastolic blood pressure.

Table 2 Comparison of diameter, Vm, blood flow volume of OA/ICA and vessel density between the affected and the contralateral healthy side in the patients with NAION

\begin{tabular}{|c|c|c|c|}
\hline Groups & NAION eye & Normal eye & $P$ \\
\hline OA diameter (mm) & $1.39 \pm 0.12$ & $1.41 \pm 0.14$ & $0.054^{* * *}$ \\
\hline $\mathrm{OA} \mathrm{Vm}(\mathrm{cm} / \mathrm{s})$ & $11.56 \pm 2.77$ & $12.17 \pm 2.75$ & $0.083^{\star \star \star}$ \\
\hline OA blood flow volume & $176.96 \pm 56.67$ & $193.00 \pm 61.04$ & $0.015^{\star \star \star}$ \\
\hline \multicolumn{4}{|l|}{$\left(\mathrm{mm}^{3} / \mathrm{s}\right)$} \\
\hline ICA diameter (mm) & $2.90 \pm 0.72$ & $3.18 \pm 0.50$ & $0.001^{* * *}$ \\
\hline ICA Vm (cm/s) & $46.04 \pm 5.83$ & $45.97 \pm 5.52$ & $0.938^{\star \star *}$ \\
\hline ICA blood flow volume & $3097.75 \pm 1131.55$ & $3691.07 \pm 1076.24$ & $<0.001^{* * *}$ \\
\hline \multicolumn{4}{|l|}{$\left(\mathrm{mm}^{3} / \mathrm{s}\right)$} \\
\hline Peripapillary vessel density & $48.90 \pm 6.02$ & $56.33 \pm 2.58$ & $0.001^{* * *}$ \\
\hline \multicolumn{4}{|l|}{ (\% area) } \\
\hline Optic disc vessel density & $45.77 \pm 4.91$ & $54.75 \pm 2.39$ & $0.001^{* * *}$ \\
\hline (\% area) & & & \\
\hline
\end{tabular}

Independent-samples t-test ${ }^{*}, \mathrm{X} 2$ test ${ }^{* *}$, Paired- samples t-test ${ }^{* * *}$

$N A I O N$ non-arteritic anterior ischemic optic neuropathy, $O A$ ophthalmic artery, $V m$ mean blood flow velocity, ICA internal carotid artery

Table 3 Comparison of diameter, Vm, blood flow volume of OA/ICA and vessel density between the affected side of the patients with NAION and the controls

\begin{tabular}{llrl}
\hline Groups & NAION eye & Controls & $P$ \\
\hline OA diameter $(\mathrm{mm})$ & $1.39 \pm 0.12$ & $1.43 \pm 0.15$ & $0.179^{*}$ \\
OA Vm (cm/s) & $11.56 \pm 2.77$ & $12.52 \pm 1.45$ & $0.052^{*}$ \\
OA blood flow volume & $176.96 \pm 56.67$ & $200.79 \pm 41.65$ & $0.031^{*}$ \\
$\left(\mathrm{~mm}^{3} / \mathrm{s}\right)$ & & & $0.001^{*}$ \\
ICA diameter $(\mathrm{mm})$ & $2.90 \pm 0.72$ & $3.36 \pm 0.51$ & $0.364^{*}$ \\
ICA Vm (cm/s) & $46.04 \pm 5.83$ & $47.03 \pm 3.92$ & $<0.001^{*}$
\end{tabular}


$\left(\mathrm{mm}^{3} / \mathrm{s}\right)$

Peripapillary vessel density

(\% area)

Optic disc vessel density

(\% area)

Independent-samples t-test ${ }^{*}, \mathrm{X} 2$ test ${ }^{* *}$, Paired- samples t-test ***

NAION non-arteritic anterior ischemic optic neuropathy, $O A$ ophthalmic artery, $V m$ mean blood flow velocity, ICA internal carotid artery

Table 4 Correlation analyses between vessel density and the blood flow volume (affected side of the patients with NAION)

\begin{tabular}{lcccr}
\hline Parameters & \multicolumn{2}{c}{ Peripapillary vessel density } & \multicolumn{2}{c}{ Optic disc vessel density } \\
\cline { 2 - 5 } & $r$ & $P$ & $r$ & $P$ \\
\hline OA blood flow volume & 0.516 & 0.004 & 0.631 & $<0.001$ \\
ICA blood flow volume & 0.527 & 0.003 & 0.578 & 0.001 \\
\hline
\end{tabular}

$O A$ ophthalmic artery, ICA internal carotid artery

\section{Discussion}

NAION is believed to be the consequence of acute ischaemia of the optic nerve head[14], but the exact pathogenesis of NAION remains a matter of debate. As mentioned earlier, previous studies suggested that the alterations in OA and ICA blood flow were often involved in ocular ischemic diseases[4,7,8]. In the course of studying NAION, the changes of blood flow volume in OA and ICS have always been our concern. As we have known, the change of blood flow velocity has always been one of the indispensable indexes to be evaluated. However, with so many uncertainties affecting changes in blood flow velocity, researchers are sometimes even led astray. Therefore, the present study attempted to establish a link between blood flow volume alterations in retrobulbar artery and ICA and the pathogenesis of NAION.

With the development of medical imaging technology and examination methods, more and more research tools are available to us. CDI is an ultrasonic imaging modality that provides a display of the real-time direction and velocity of the blood flow imposed over a conventional gray-scale B-mode ultrasound image [15]. Previous studies found that patients with NAION 
showed reduced circulation velocity in the retrobulbar vessels and decreased velocities of blood cells in the capillaries of the optic nerve head by means of CDI or laser Doppler velocimetry [4, $5,16,17]$, but the resolution of CDI is insufficient to provide reliable volumetric flow measurements. Fortunately, it's possible to detect the diameter of blood vessels using CTA precisely. Nowadays, CTA has been recommended as a safe and non-invasive tool for the assessment of OA, carotid, coronary, and renal artery stenosis due to its ability to achieve clear image reconstruction [18-20]. With the data obtained from CDI and TCD, blood flow volume of ICA and OA can be calculated indirectly according to $\mathrm{Vm}$ and blood vessel diameter.

In our study, we found the diameter of the ipsilateral ICA distinctly narrowed in the affected side of patients suffering from NAION, compared with the contralateral healthy side or with the controls. Accordingly, the blood flow volume of ICA in the patients with NAION significantly decreased, although there was no significant difference in Vm of ICA between groups. As a rule, the flow velocity increases as a result of constriction or narrowing of a vessel lumen [21]. However, Considering that blood is a non-Newtonian fluid, the viscosity of plasma and the elasticity of the vessel wall both have an effect on the flow velocity[12]. According to the law of conservation of mass, when blood flows through a certain section of blood vessel, the blood flow volume should be a fixed value, so we can obtain the instantaneous blood flow volume by measuring the diameter of the vessel and the blood flow velocity. We speculated that when NAION occurred, decreased intravascular blood flow was caused by narrowing ICA diameter, which ultimately resulted in significantly reduced blood perfusion to the microvessels in the downstream region. However, previous studies proved that the degree of ICA stenosis had no significant differences between the NAION group and the controls [22-24]. This discrepancy might be explained by the different measurement locations selected. In previous studies, the entire ICA vessel was taken as the research object, while in this study, only the siphon segment of ICA was selected as the measurement object. We designed it this way because ICAs are considered to be a very specific part of the ICA vascular pathway, and the tortuous configuration of ICAS is crucial to diseases related to turbulent circulation [25], especially to ocular ischemic diseases.

In addition, we noted that the diameter of $\mathrm{OA}$ and the $\mathrm{Vm}$ of OA were not significantly different in NAION patients compared with the contralateral healthy side and the controls, but the blood flow volume of OA in NAION patients was significantly reduced compared with the other two 
groups. Combined with the measurement results of ICAS, we believed that the blood flow velocity alone is not a reliable parameter in the study of NAION, while the blood flow volume seems to be more reliable, which can accurately reflect the status of blood perfusion. Based on these findings, it was reasonable to assume that evaluating blood flow is more important in ischemic diseases than focusing solely on blood flow velocity.

To further investigate the relationship between blood flow volume alterations of OA and ICA and the developing of NAION, we measured peripapillary and optic disc vessel density. Moreover, the correlation between vascular density and blood flow volume were analyzed. Currently, laser speckle flowgraphy (LSFG) and OCTA have both been developed as non-invasive methods for evaluating ONH blood flow, and they are both reliable and repeatable techniques [26-29]. LSFG is a promising tool for the evaluation of microvascular lesions in the retina and $\mathrm{ONH}$, and further clinical validation is required $[25,26]$. In this study, OCTA was used to quantitatively evaluate the microcirculation changes of ONH in patients with NAION. The reason for this choice was that, on the one hand, previous studies have described potential efficacy for OCTA in the evaluation of NAION through quantified detecting reduced ONH perfusion [30-32], and on the other hand, it was reported that vessel densities at the peripapillary retinal and optic disc were closely related to retinal nerve fiber damage and visual field loss [33,34], and OCTA is able to display both structural and blood flow information to help quantify the $\mathrm{ONH}$ perfusion and retinal vasculature $[35,36]$. In our study, the result showed that peripapillary and optic disc vessel density all significantly decreased in the affected side of NAION patients compared with the contralateral healthy side and the control group, which is consistent with previous studies [30,31]. Furthermore, we found both peripapillary vessel density and optic disc vessel density were significantly positive correlated with blood flow volume of ICA and OA in the affected side of patients with NAION. Peripapillary and optic disc vessel density can be used as a reliable and stable indicator for the changes in peripheral vascular status caused by OA and ICA blood flow volume alterations. This finding further strengthened the evidence that changes in blood flow volume of retrobulbar artery and ICA play an important role in the pathogenesis of NAION.

There are several limitations in our study. Firstly, the selection bias of participants might potentially affect the results. To minimize this bias, we designed age- and sex-matched controls, and attempted to make sure that the distribution of vascular disease characteristics was matched 
between patients and controls. However, Since systemic diseases are more common in the study group (although the differences of prevalence of systemic diseases between patients and controls were not statistically significant), it was still difficult to select a control group on a per- patient basis during the recruitment process. Larger scale studies with better case-control matching would be required. Another limitation of this study is that the blood flow volume obtained here was only an approximation, which was calculated based on a formula rather than directly detected by an inspection equipment. Further prospective blood flow volume studies with more accurate calculations or detection methods are necessary to confirm the present conclusions in study of NAION patients.

\section{Conclusions}

In summary, the reduction of blood flow volume was more prominent in OA and ICA than decrease of Vm in patients with NAION. This finding may imply a predominant affection of retrobulbar artery and ICA blood flow volume alterations in the pathogenesis of NAION.

\section{Abbreviations}

ICA, internal carotid artery; NAION, non-arteritic anterior ischemic optic neuropathy; CTA, head-and-neck computed tomographic angiography; OA, ophthalmic artery; CDI, colour Doppler imaging; Vm, mean blood flow velocity; OCTA, optical coherence tomography angiography; ICAS, siphon region of ICA; IOP, intraocular pressure; SD-OCT, spectral-domain optical coherence tomography scans; ILM, inner limiting membrane; NFL, nerve fiber layer; BP, blood pressure; SBP, systolic blood pressure; DBP, diastolic blood pressure; LSFG, laser speckle flowgraphy

\section{Acknowledgements}

Not applicable.

\section{Authors' contributions}

ZYF and HYL designed the study. ZYF contributed to the analysis of data and drafting the manuscript and revising it critically. HYL was responsible for the interpretation of data. YLW was involved in the revising of the manuscript as well as provided final approval of the version to be published.

\section{Funding}

This research was supported by "The Capital Health Research and Development of Special (2018-1-2021)". The funders play no role in the design of the study and collection, analysis, and interpretation of data and in writing the manuscript.

\section{Availability of data and materials}

All authors have agreed to share our raw data with others, we have provided it in the supplementary file. 


\section{Ethics approval and consent to participate}

The protocol of this study has been approved by the Ethics Committee of Capital Medical University Affiliated to Beijing Friendship Hospital and informed consent has been obtained from all participants.

\section{Consent for publication}

Not Applicable.

\section{Competing interests}

The authors declare that they have no competing interests.

\section{References}

1. Hata M, Oishi A, Muraoka Y, Miyamoto K, Kawai K, Yokota S, et al. Structural and functional analyses in nonarteritic anterior ischemic optic neuropathy: optical coherence tomography angiography study. J Neuroophthalmol. 2017; 37: $140-8$.

2. Gaier ED, Torun N. The enigma of nonarteritic anterior ischemic optic neuropathy: an update for the comprehensive ophthalmologist. Curr Opin Ophthalmol. 2016; 27: 498-504.

3. Kaup M, Plange N, Arend KO, Remky A. Retrobulbar hemodynamics in nonarteritic anterior ischemic optic neuropathy. Br J Ophthalmol. 2006; 90:1350-3.

4. Sanjari MS, Falavarjani KG, Mehrabani M, Ghiasian L, Zamani B. Retrobulbar haemodynamics and carotid wall thickness in patients with non-arteritic anterior ischaemic optic neuropathy. $\mathrm{Br} J$ Ophthalmol. 2009; 93:638-40.

5. Zhu WH, Cui MY, Yao FJ, Liao RD, Liu L. Retrobulbar and common carotid artery haemodynamics and carotid wall thickness in patients with non-arteritic anterior ischemic optic neuropathy. Graefes Arch Clin Exp Ophthalmol. 2014; 252:1141-6.

6. Arnold AC. The 14th Hoyt lecture: ischemic optic neuropathy: the evolving profile,1966-2015. J Neuroophthalmol. 2016; 36: 208-15.

7. Brown GC. Anterior ischemic optic neuropathy occurring in association with carotid artery obstruction. J Clin Neuroophthalmol. 1986; 6:39-42.

8. Barkana Y, Harris A, Hefez L, Zaritski M, Chen D, Avni I. Unrecordable pulsatile ocular blood flow may signify severe stenosis of the ipsilateral internal carotid artery. Br J Ophthalmol. 2003; 87: 1478-80.

9. Singh R, Tubbs RS. Effect of cervical siphon of external and internal carotid arteries. J Craniofac Surg. 2017; 28: $1857-60$

10. Hayreh SS. Ischemic optic neuropathy. Prog Retin Eye Res. 2009; 28:34-62.

11. Hayreh SS, Zimmerman MB. Nonarteritic anterior ischemic optic neuropathy: natural history of visual outcome. Ophthalmology. 2008;115:298-305.

12. Fu ZY, Li HY, Wang W, Wang YL. Research on association of the diameter of the internal carotid artery siphon and nonarteritic anterior ischaemic optic neuropathy. J Ophthalmol. 2019; 2019:7910602.

13. Fu ZY, Li HY, Wang YL. Imageology of internal carotid artery siphon in nonarteritic anterior ischaemic optic neuropathy. Int J Ophthalmol. 2020; 13: 1941-7.

14. Mayes EW, Cole ED, Dang S, Novais EA, Vuong L, Mendoza-Santiesteban C, et al. Optical coherence tomography angiography in nonarteritic anterior ischemic optic neuropathy. J Neuroophthalmol. 2017; 37: 358-64.

15. Quaranta L, Harris A, Donato F, Cassamali M, Semeraro F, Nascimbeni G, et al. Color Doppler imaging of ophthalmic artery blood flow velocity: A study of repeatability and agreement. Ophthalmology. 1997; 104:653-8.

16. Leiba H, Rachmiel R, Harris A, Kagemann L, Pollack A, Zalish M. Optic nerve head blood flow measurements in non-arteritic anterior ischaemic optic neuropathy. Eye (Lond). 2000; 14:828-33.

17. Collignon-Robe NJ, Feke GT, Rizzo JF. Optic nerve head circulation in nonarteritic anterior ischemic optic neuropathy and optic neuritis. Ophthalmology. 2004; 111:1663-72.

18. Dix JE, Evans AJ, Kallmes DF, Sobel AH, Phillips CD. Accuracy and precision of CT angiography in a model of carotid artery bifurcation stenosis. Am J Neuroradiol. 1997;18:409-15.

19. Yamada M, Jinzaki M, Kuribayashi S, Sato K, Tanami Y, Yun S, et al. Quantitative evaluation of coronary arterial stenosis using 16-slice multidetector-row computed tomography: preliminary evaluation of phantom study. J Comput Assist Tomogr. 2005; 29:382-6. 
20. Erdogmus S, Govsa F. Accurate course and relationships of the intraorbital part of the ophthalmic artery in the sagittal plane. Minim Invasive Neurosurg. 2007; 50:202-8.

21. Kagemann L, Harris A, Chung HS, Costa VP, Garzozi HJ. Basics and limitations of Color Doppler imaging. In: Pillunat LE, Harris A, Anderson DR, Greve EL, eds. Current concepts on ocular blood flow in glaucoma. The Hague, Netherlands: Kugler; 1999;103-10.

22. Fry CL, Carter JE, Kanter MC, Tegeler CH, Tuley MR. Anterior ischemic optic neuropathy is not associated with carotid artery atherosclerosis. Stroke. 1993; 24:539-42.

23. Muller M, Kessler C, Wessel K, Mehdorn E, Kompf D. Low-tension glaucoma: a comparative study with retinal ischemic syndromes and anterior ischemic optic neuropathy. Ophthalmic Surg. 1993; 24: 835-8.

24. McCullough HK, Reinert CG, Hynan LS, Albiston CL, Inman MH, Boyd PI, et al. Ocular findings as predictors of carotid artery occlusive disease: is carotid imaging justified? J Vasc Surg 2004; 40:279-86.

25. Zenteno M, Vinuela F, Moscote-Salazar LR, Alvis-Miranda H, Zavaleta R, Flores A, et al. Clinical implications of internal carotid artery tortuosity, kinking and coiling: a systematic review. Rom Neurosurg 2014; 21:50-9.

26. Fondi K, Bata AM, Luft N, Witkowska KJ, Werkmeister RM, Schmidl D, et al. Evaluation of flicker induced hyperemia in the retina and optic nerve head measured by laser speckle flowgraphy. PLoS ONE. 2018;13:e0207525.

27. Kunikata H, Nakazawa T. Recent clinical applications of laser speckle flowgraphy in eyes with retinal disease. Asia-Pacific J ophthalmol. 2016;5:151-8.

28. Suwan Y, Geyman LS, Fard MA, Tantraworasin A, Chui TY, Rosen RB, et al. Peripapillary perfused capillary density in exfoliation syndrome and exfoliation glaucoma versus POAG and healthy controls: An OCTA study. Asia-Pacific J ophthalmol. 2018;7:84-9.

29. SongY, Min JY, Mao L, Gong YY. Microvasculature dropout detected by the optical coherence tomography angiography in nonarteritic anterior ischemic optic neuropathy. Lasers Sur Med. 2018;50:194-201.

30. Sharma S, Ang M, Najjar RP, Sng C, Cheung CY, Rukmini AV, et al. Optical coherence tomography angiography in acute non-arteritic anterior ischaemic optic neuropathy. Br J Ophthalmol. 2017;101:1045-51.

31. Rebolleda G, Diez-Alvarez L, Garcia Marin Y, de Juan V, Munoz-Negrete FJ. Reduction of peripapillary vessel density by optical coherence tomography angiography from the acute to the atrophic stage in non-arteritic anterior ischaemic optic neuropathy. Ophthalmologica. 2018;240:191-9.

32. Higashiyama T, Ichiyama Y, Muraki S, Nishida Y, Ohji M. Optical coherence tomography angiography in a patient with optic atrophy after non-arteritic anterior ischaemic optic neuropathy. Neuroophthalmology. 2016;40:146-9.

33. Yarmohammadi A, Zangwill LM, Diniz-Filho A, Saunders LJ, Suh MH, Wu Z, et al. Peripapillary and macular vessel density in patients with glaucoma and single-hemifield visual field defect. Ophthalmology. 2017;124:709-19.

34. Wang X, Jiang C, Ko T, Kong X, Yu X, Min W, et al. Correlation between optic disc perfusion and glaucomatous severity in patients with openangle glaucoma: an optical coherence tomography angiography study. Graefes Arch Clin Exp Ophthalmol. 2015;253:1557-64.

35. Jia Y, Tan O, Tokayer J, Potsaid B, Wang Y, Liu JJ, et al. Split-spectrum amplitude-decorrelation angiography with optical coherence tomography. Opt Express. 2012; 20: 4710-25.

36. Mo S, Phillips E, Krawitz BD, Garg R, Salim S, Geyman LS, et al.. Visualization of radial peripapillary capillaries using optical coherence tomography angiography: The effect of image averaging. PLoS ONE. 2017;12:e0169385. 
Figures

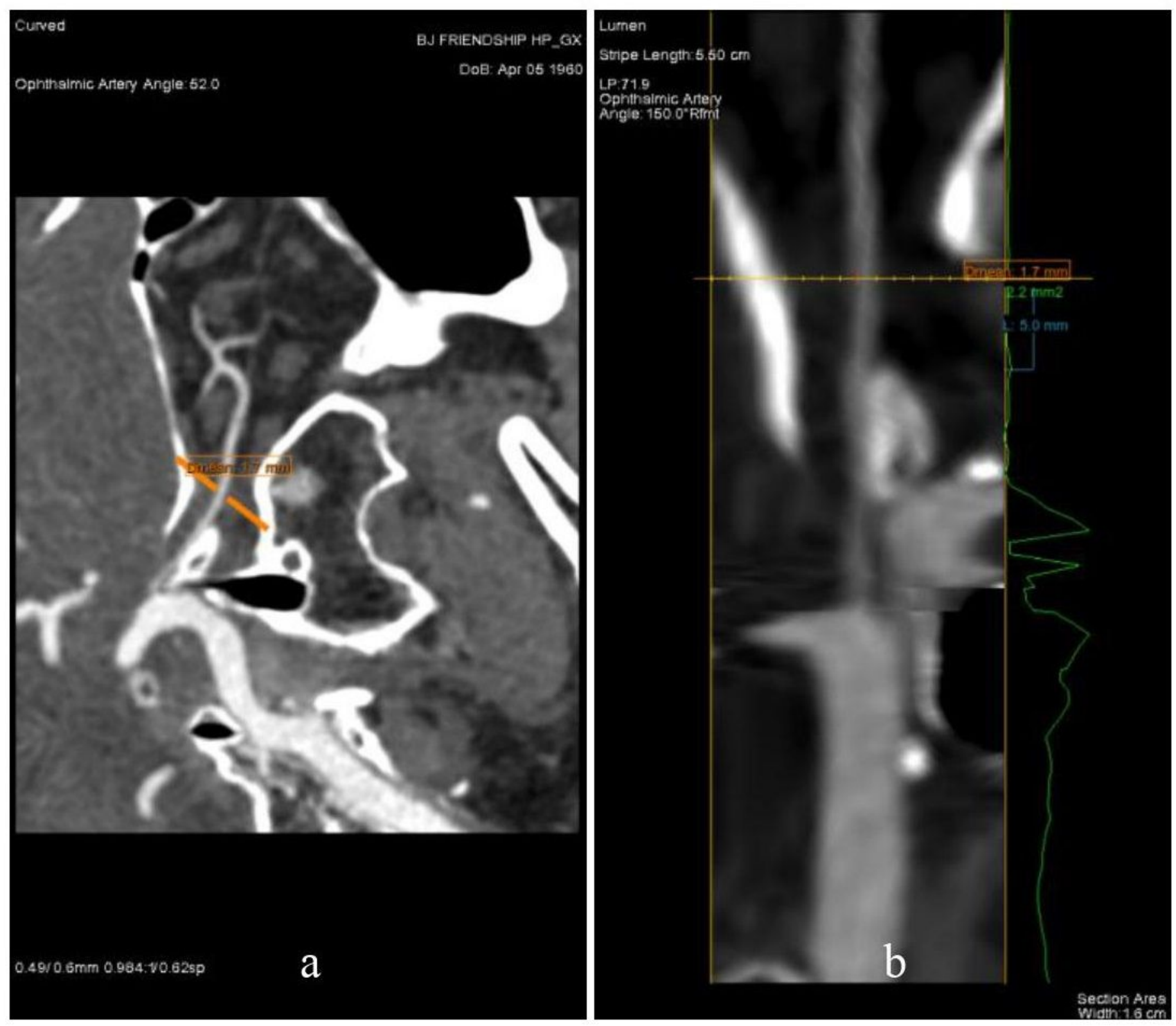

Figure 1

Head-and-neck CTA inspection. Showed the diameter of OA ( $a, b$ ) 

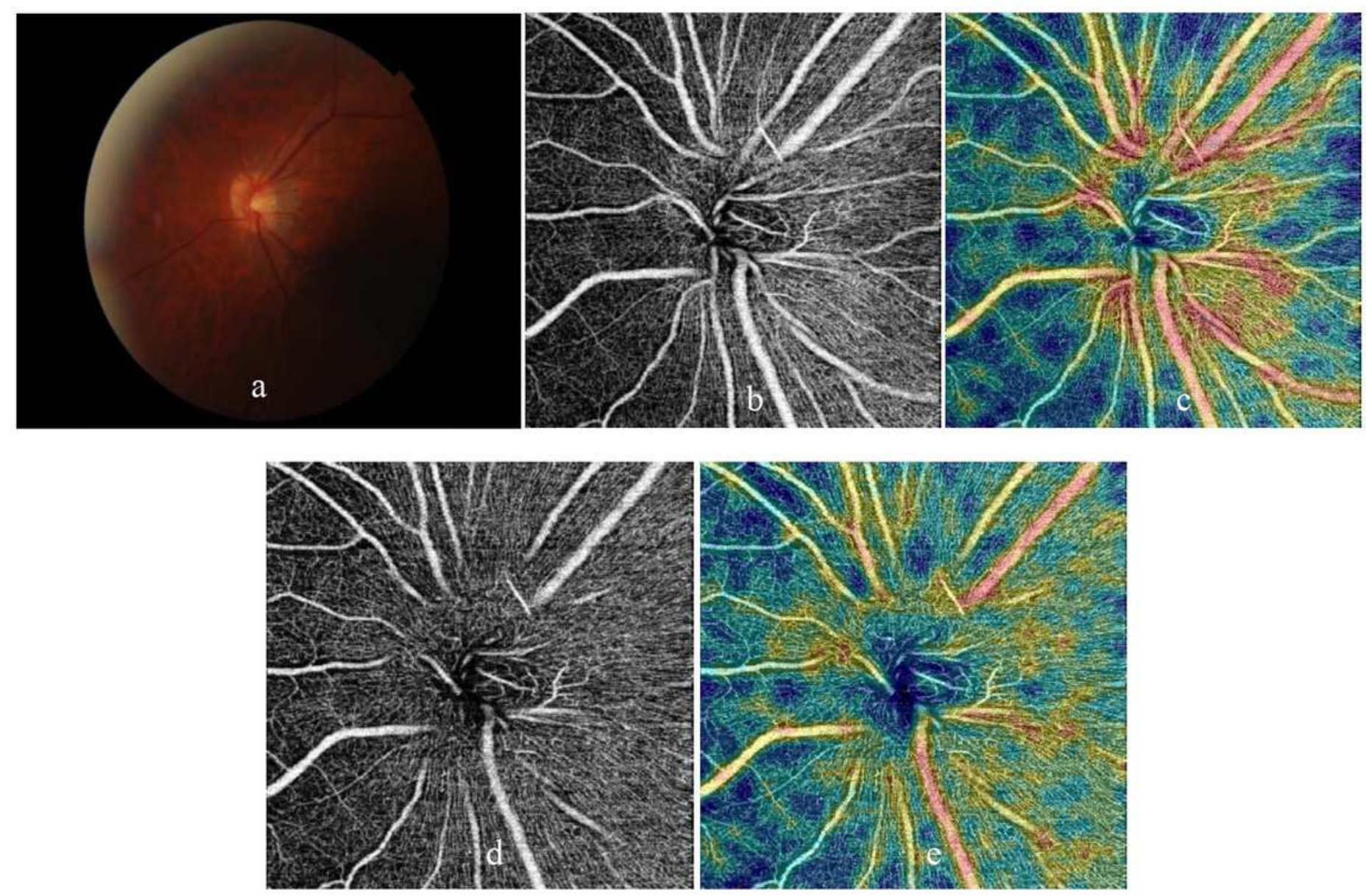

\section{Figure 2}

OCTA examinations of peripapillary and optic disc vessel density of a control subject (left eye). Color fundus photograph (a),vessel density in the peripapillary retina on nerve head mode (b, $c)$ and optic disc on RPC mode ( $d, e)$ were included (b, d: images in gray scale, c, e: color-coded images) 

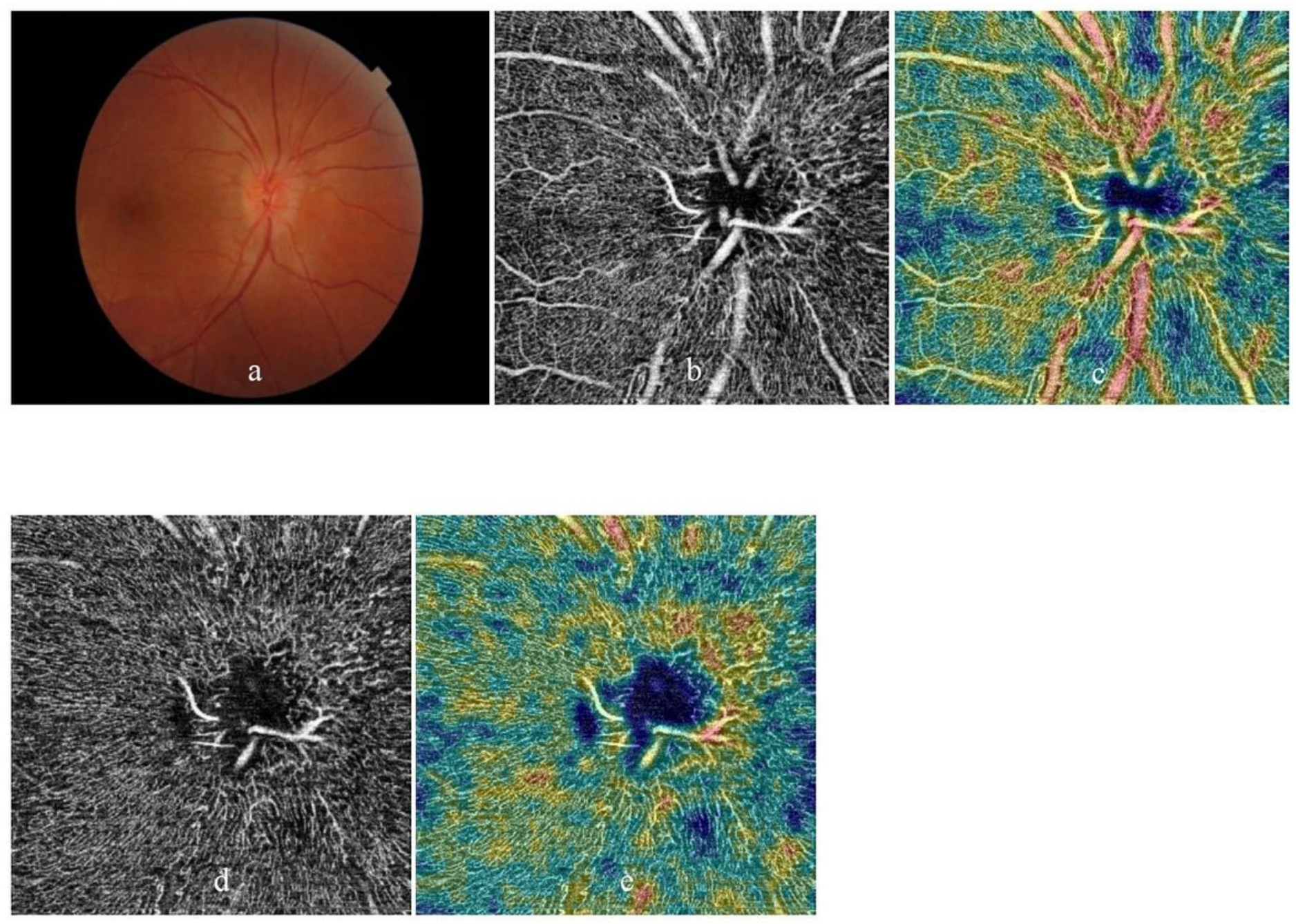

\section{Figure 3}

OCTA examinations of peripapillary and optic disc vessel density of a NAION patient (affected left eye ). Color fundus photograph (a),vessel density in the peripapillary retina on nerve head mode (b, c) and optic disc on RPC mode ( $d, e)$ were included (b, d: images in gray scale, $c$, e: color-coded images)

\section{Supplementary Files}

This is a list of supplementary files associated with this preprint. Click to download.

- supplementalfile.docx 